\title{
Colitis ulcerativa para no gastroenterólogos
}

\author{
Ulcerative colitis for non-gastroenterologists
}

Nicolás Zuluaga-Arbeláez', Elsy Cristina Sierra-Vargas ${ }^{2 \text { CvLAC }}$, Juan Camilo Díaz-Coronado ${ }^{3 \text { CvLAC, }}$
Luis Gonzalo Guevara-Casallas

Fecha correspondencia:

Recibido: julio 2 de 2020.

Revisado: septiembre 18 de 2020.

Aceptado: octubre 20 de 2020.

Forma de citar:

Zuluaga-Arbeláez N, Sierra-Vargas

EC, Díaz-Coronado JC, Guevara-

Casalla LG. Colitis ulcerativa para

no gastroenterólogos. Rev CES

Med. 2020; 34(3): 188-197.

Open access

(C) Derecho de autor

Licencia creative commons

Etica de publicaciones

Revisión por pares

Gestión por Open Journal System

DOl: http://dx.doi.org/10.21615/

cesmedicina.34.3.2

ISSN 0120-8705

e-ISSN 2215-9177

Sobre los autores:

1. Residente Medicina Interna.

Universidad CES.

2. Especialista en Medicina

Interna. Universidad de Antioquia.

Comparte

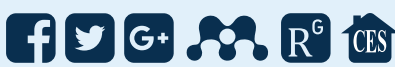

\section{Resumen}

La colitis ulcerativa es una enfermedad intestinal inflamatoria crónica, de etiología idiopática y fisiopatología multifactorial, caracterizada por la inflamación de la mucosa rectal, con extensión proximal, continua y simétrica a través del colon. Su diagnóstico se establece a partir de los hallazgos clínicos, endoscópicos e histológicos. La meta terapéutica es lograr la remisión clínica y endoscópica, reducir el riesgo de colectomía, cáncer colorrectal y mejorar la calidad de vida de los pacientes. Se realizó una búsqueda de artículos científicos y se exponen los aspectos más relevantes y actualizados sobre la enfermedad; con el objetivo de proporcionar elementos para su abordaje diagnóstico y terapéutico.

Palabras clave: Colitis ulcerosa; Enfermedades intestinales; Diagnóstico, Terapéutica.

\begin{abstract}
Ulcerative colitis is a chronic inflammatory bowel disease of idiopathic etiology and multifactorial pathophysiology. It is characterized by inflammation of the rectal mucosa with proximal, continuous and symmetrical extension through the colon. Its diagnosis is established based on clinical, endoscopic and histological findings. The therapeutic goal is to achieve clinical and endoscopic remission, reduce the risk of colectomy, colorectal cancer and improve quality of life of patients. A search for scientific articles was carried out, achieving this narrative review of the literature where the most relevant and updated aspects of the disease are exposed; with the objective of providing elements for the diagnostic and therapeutic approach of the disease.
\end{abstract}

Keywords: Ulcerative colitis; Intestinal diseases; Diagnosis; Therapeutics.

\section{Introducción}

La colitis ulcerativa es la forma más común de enfermedad inflamatoria intestinal en el mundo $(1,2)$. Históricamente, ha sido considerada como idiopática y se caracteriza por la inflamación crónica de la mucosa del colon, con especial afectación a la mucosa rectal y con extensión proximal, continua y simétrica en ausencia de granulomas (1). Con el objetivo de exponer los aspectos más relevantes y actualizados sobre esta enfermedad se realizó una búsqueda de artículos científicos en PubMed, EMBASE, Google Scholar y SciELO, logrando esta revisión narrativa. 
Septiembre - diciembre de 2020 - Pág 189

3. Especialista en Medicina Interna, Magister en epidemiología. Universidad CES.

4. Médico, Especialista en Medicina Interna y Gastroenterología.
Los síntomas más frecuentes son diarrea con moco y sangre, dolor abdominal, urgencia y tenesmo rectal. No obstante, hasta el $10 \%$ de los pacientes con proctitis/ proctosigmoiditis tiene estreñimiento paradójico, por enlentecimiento del tránsito intestinal proximal debido al proceso inflamatorio.

\section{Epidemiología}

La prevalencia mundial oscila entre 0,008 a 0,24 \% y la incidencia entre 0,001 \% a $0,02 \%$, ambas en incremento durante los últimos años, explicado por un aumento en la detección diagnóstica y mayor exposición ambiental a factores de riesgo (cuadro 1) (1,2).

Con los avances en las técnicas diagnósticas y terapias médicas se ha logrado disminuir su mortalidad (3). Sin embargo, la morbilidad que confiere afecta grandemente la calidad de vida $(4,5)$ y aumenta de forma significativa los costos para el sistema de salud (6).

Cuadro 1. Principales factores de riesgo para colitis ulcerativa

\begin{tabular}{ll}
\hline Factor de riesgo & Descripción \\
\hline Edad & Distribución bimodal: 15-30 años y 50-70 años. \\
\hline Género & La incidencia anual por género es mayor en hombres. \\
\hline Historia familiar & $\begin{array}{l}\text { El riesgo de enfermedad inflamatoria intestinal aumenta } \\
\text { significativamente, hasta en un 12 \%, si hay antecedentes familiares. }\end{array}$ \\
\hline Dieta & Mayor riesgo asociado al consumo de grasas mono y poliinsaturadas. \\
\hline Microbiota & Disbiosis del microbiota intestinal. \\
\hline Infección gastrointestinal & La gastroenteritis infecciosa aumenta el riesgo de desarrollo de Ell. \\
\hline Medicamentos & $\begin{array}{l}\text { Antiinflamatorios no esteroideos, anticonceptivos orales y terapia de } \\
\text { reemplazo hormonal. }\end{array}$ \\
\hline
\end{tabular}

Adaptado de Referencia (4)

\section{Fisiopatología}

Existen varias hipótesis sobre la patogénesis de la enfermedad. Dentro de las más aceptadas está la pérdida de la regulación tridireccional entre la microbiota, las células del epitelio intestinal y el sistema inmune propio de la mucosa, que en un individuo genéticamente susceptible y en presencia de factores de riesgo alterará este equilibrio, produciendo un ambiente altamente inflamatorio. La microbiota despierta especial interés porque es esencial para el correcto desarrollo del sistema inmune intestinal, provee elementos nutricionales a las células del intestino y modula el metabolismo energético. Cuando se altera la homeostasis en el epitelio intestinal, la misma flora comensal puede actuar como patógeno dando lugar a la liberación de citoquinas proinflamatorias (p.e. FNT, IL-1 $\beta$, IFN- $\gamma$ ). Se describe también una alteración cuantitativa o cualitativa de la microbiota (disbiosis). La pérdida de bacterias protectoras (p.e. Firmicutes y Bacteroidetes) es importante puesto que producen ácidos grasos de cadena corta que sirven de sustrato energético para los colonocitos; además, su ausencia favorece la proliferación de bacterias patógenas (7).

\section{Presentación clínica}

El compromiso intestinal se distribuye anatómicamente así: 30 a 60 \% se presentarán con proctitis, 16 a $45 \%$ con colitis izquierda y 15 a $35 \%$ con colitis extensa. El inicio de la enfermedad es insidioso y los síntomas pueden estar presentes por semanas o meses antes de consultar al médico (8). Los síntomas más frecuentes son diarrea con moco y sangre, dolor abdominal, urgencia y tenesmo rectal. No obstante, hasta el $10 \%$ de los pacientes con proctitis/proctosigmoiditis tiene estreñimiento paradójico, por enlentecimiento del tránsito intestinal proximal debido al proceso inflamatorio (8). 
El curso natural de la enfermedad supone periodos alternantes de recurrencia y remisión en el $90 \%$ de los pacientes, aún después de 25 años del diagnóstico.
Cuando el compromiso de la mucosa intestinal es extenso suele haber diarrea sanguinolenta abundante, pérdida de peso, fiebre y dolor abdominal marcado, que llevan a malnutrición, hipoalbuminemia y anemia ferropénica (1). Con la palpación abdominal puede encontrarse dolor difuso o circunscrito al segmento intestinal afectado, defensa abdominal e incluso signos de irritación peritoneal si coexisten complicaciones. El tacto rectal puede revelar estigmas de sangrado (8).

El curso natural de la enfermedad supone periodos alternantes de recurrencia y remisión en el $90 \%$ de los pacientes, aún después de 25 años del diagnóstico. La actividad persistente solo llega al $1 \%$ y $0,1 \%$ tras cinco y 25 años, respectivamente (9). Las manifestaciones extraintestinales se presentan hasta en un tercio de los pacientes con colitis ulcerativa y pueden preceder los síntomas gastrointestinales (cuadro 2) (10).

Cuadro 2. Manifestaciones extraintestinales

\begin{tabular}{llc}
\hline \multicolumn{1}{c}{ Órgano/sistema } & \multicolumn{1}{c}{ Manifestación } & Prevalencia (\%) \\
\hline \multirow{2}{*}{ Osteoarticular } & Artropatía periférica & 21 \\
\cline { 2 - 3 } & Compromiso axial (espondilitis o sacroileitis) & 2 \\
\hline \multirow{4}{*}{ Piel } & Eritema nodoso & $10-20$ \\
\cline { 2 - 3 } & Pioderma gangrenoso & 2 \\
\cline { 2 - 3 } & Estomatitis aftosa & 4 \\
\hline \multirow{3}{*}{ Ojos } & Síndrome de Sweet & $<1$ \\
& Uveitis & 4 \\
\hline Hepatobiliar & Episcleritis & $<1$ \\
\cline { 2 - 3 } & Escleritis & 4 \\
\hline
\end{tabular}

\section{Complicaciones}

Colitis fulminante y megacolon tóxico: la presencia de más de 10 deposiciones al día, sangrado rectal continuo y compromiso sistémico caracterizan clínicamente a la colitis fulminante, condición de riesgo para desarrollar megacolon tóxico y definido radiológicamente por un diámetro colónico transverso $\geq 6 \mathrm{~cm}$ o un diámetro cecal $>9$ $\mathrm{cm}$. Debe considerarse infección por $C$. difficile o citomegalovirus como precipitantes $(11,12)$.

Perforación espontanea: descrita en alrededor del $8 \%$ de los casos. Se manifiesta con dolor abdominal intenso y signos de irritación peritoneal $(11,12)$.

Estenosis: descrita hasta en el $10 \%$ de pacientes. Obliga a descartar cáncer mediante evaluación endoscópica y biopsia (13).

Cáncer colorrectal: se reporta un aumento en su incidencia luego de 8-10 años del diagnóstico de colitis ulcerativa. Recomendándose iniciar tamizaje a los ocho años de evolución y repetirse en intervalos de uno a tres años, según el riesgo individual (4). 


\section{Diagnóstico}

La enfermedad se sospecha por la clínica descrita y se confirma con los hallazgos endoscópicos e histopatológicos que permitirán diferenciar esta enfermedad de otras condiciones que tienen una presentación similar (8) (cuadro 3).

Cuadro 3. Principales diagnósticos diferenciales

\begin{tabular}{l} 
Colitis infecciosa (bacterias, virus, hongos, micobacterias, Clostridioides difficile) \\
Colitis isquémica \\
\hline Colitis segmentaria asociada a diverticulitis \\
Colitis/proctitis inducida por radiación \\
Colitis inducida por medicamentos \\
Enfermedad de Crohn \\
Enfermedades de transmisión sexual causantes de proctitis (sífilis, herpes, clamidia, \\
gonorrea y linfogranuloma venéreo)
\end{tabular}

La ileocolonoscopia es indispensable para realizar el diagnóstico, porque permite evaluar la extensión y severidad de la enfermedad y descartar compromiso del íleon distal, en cuyo caso habría que considerar enfermedad de Crohn.

\section{Estudio endoscópico}

La ileocolonoscopia es indispensable para realizar el diagnóstico, porque permite evaluar la extensión y severidad de la enfermedad y descartar compromiso del íleon distal, en cuyo caso habría que considerar enfermedad de Crohn (4). Cuando hay marcadores clínicos de gravedad (más de seis deposiciones sanguinolentas por día, caída de la hemoglobina, elevación marcada de reactantes), debe realizarse rectosigmoidoscopia con mínima insuflación para prevenir el riesgo de perforación (14).

Típicamente, se evidencia inflamación en la mucosa que inicia a nivel rectal y que, de manera simétrica, circunferencial y confluente, asciende por el colon en dirección proximal (8). Si bien los hallazgos endoscópicos dependen de la gravedad (figura 1), existe clara diferenciación entre la mucosa no inflamada e inflamada, siendo claves el eritema, la pérdida del patrón vascular normal y la friabilidad del tejido.

Hasta el $75 \%$ de los pacientes con colitis ulcerativa del lado izquierdo presenta un área aislada de inflamación peri-apendicular, conocida como parche cecal. El $20 \%$ de los individuos con pancolitis tiene compromiso ileal (conocido en inglés como backwash ileitis) que, a diferencia del patrón parcheado y transmural de la enfermedad de Crohn, es continuo y solo afecta la mucosa $(1,8)$.

La esofagogastroduodenoscopia solo está indicada si hay compromiso ileal o presencia de síntomas gastrointestinales superiores, buscando descartar enfermedad de Crohn (13).
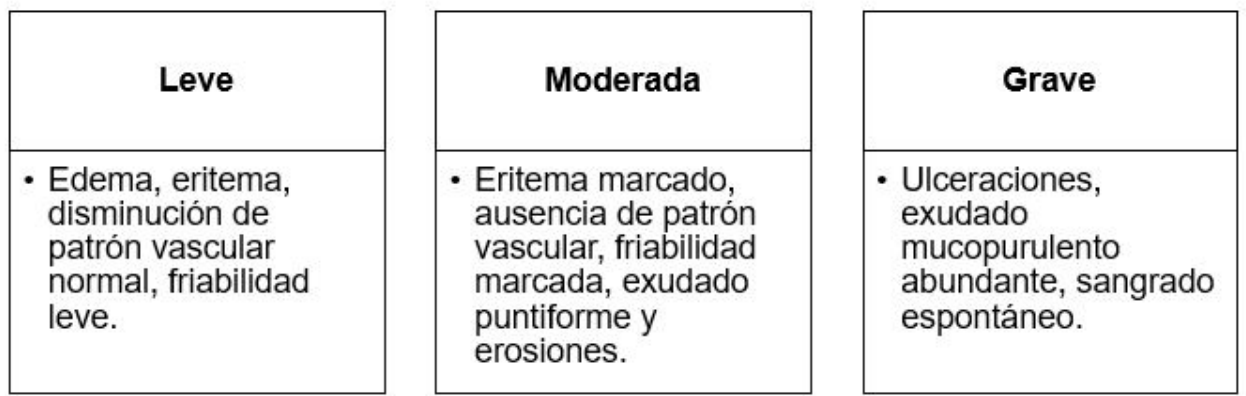

Figura 1. Hallazgos endoscópicos según la gravedad de la colitis ulcerativa Adaptado de referencia (13) 
Para que el diagnóstico sea confiable se deben tomar al menos dos biopsias de cada una de las de seis áreas recomendadas (íleon terminal, colon ascendente, transverso, descendente, sigmoide y recto).

\section{Estudio histológico}

Para que el diagnóstico sea confiable se deben tomar al menos dos biopsias de cada una de las de seis áreas recomendadas (íleon terminal, colon ascendente, transverso, descendente, sigmoide y recto), incluida la mucosa de apariencia normal, ya que, en ocasiones, los cambios inflamatorios pueden ser evidentes solo histológicamente (4).

Característicamente, los hallazgos histológicos se encuentran restringidos a la capa mucosa, con infiltrados variables en densidad y composición según actividad o etapa de remisión. Sin embargo, se puede hacer diagnóstico si presentan al menos dos de estos cuatro cambios microscópicos: disminución marcada de la densidad de las criptas, distorsión arquitectónica de las criptas, superficie irregular de la mucosa o inflamación transmucosa grave en ausencia de granulomas (15).

\section{Laboratorio}

Aunque en la enfermedad el rendimiento diagnóstico de los exámenes en materia fecal y sangre es bajo, se recomienda solicitarlos en todo individuo con diarrea sanguinolenta en quien se sospeche enfermedad inflamatoria intestinal. Se debe realizar coproscópico, coprocultivo y toxina de C. difficile. Otros estudios microbiológicos dependerán de la sospecha clínica.

La calprotectina fecal, proteína segregada por los neutrófilos, está incrementada superlativamente en condiciones inflamatorias (16). Niveles bajos hacen improbable (<1 \%) el diagnóstico, aunque si están elevados no confirma la enfermedad. Puede ser útil en el seguimiento de pacientes, ya que valores por encima de $250 \mu \mathrm{g} / \mathrm{g}$ han mostrado predecir recaída y cifras menores a $50 \mu \mathrm{g} / \mathrm{g}$ sugieren remisión (17).

La proteína C reactiva y la eritrosedimentación son marcadores sensibles de inflamación, pero poco específicos. En la colitis ulcerativa, los niveles altos de estos reactantes se correlacionan con gravedad endoscópica, riesgo de colectomía y pobre respuesta al tratamiento. No obstante, una cuarta parte de las personas con enfermedad endoscópicamente activa tienen proteína $\mathrm{C}$ reactiva normal (13).

Bajos niveles de albúmina sugieren actividad de la enfermedad y confieren mayor riesgo de colectomía y se asocian con pobre respuesta a medicamentos biológicos (4).

Debe solicitarse hemoleucograma, pues la anemia es una complicación frecuente, generalmente de origen multifactorial: sangrado agudo, ferropenia secundaria a sangrado crónico, mielosupresión por inflamación crónica o medicamentos como azatioprina, 6-mercaptopurina y sulfasalazina (1).

Por su bajo rendimiento diagnóstico los anticuerpos contra el citoplasma de neutrófilos (pANCA) y los anticuerpos anti Saccharomyces cerevisiae (ASCA) no son recomendados por ninguna sociedad científica $(13,4)$.

\section{Imágenes}

Su rendimiento es limitado para el diagnóstico de colitis ulcerativa, pero son útiles para evaluar complicaciones graves. En un primer nivel de atención, la radiografía simple de abdomen puede servir para descartar megacolon tóxico (diámetros descritos previamente) o perforación si se visualiza neumoperitoneo. La tomografía de abdomen contrastada será el estudio de elección en el paciente con sospecha de colitis ulcerativa 
complicada; además, permitirá evaluar los órganos intraabdominales y descartar diagnósticos diferenciales $(7,18)$.

\section{Extensión y gravedad}

Es indispensable determinar la extensión y gravedad de la enfermedad para el pronóstico y la toma de decisiones terapéuticas. Las guías recomiendan establecer la extensión con la clasificación endoscópica de Montreal (cuadro 4). En cuanto a la gravedad, recientemente el Colegio Americano de Gastroenterología propuso una nueva clasificación (cuadro 5), siendo la más recomendada actualmente (14).

Cuadro 4. Clasificación de Montreal que determina la extensión de la colitis ulcerativa

\begin{tabular}{cl}
\hline Extensión & Descripción anatómica \\
\hline E1 & Inflamación limitada al recto \\
\hline E2 & Inflamación limitada a la porción colorrectal distal al ángulo esplénico \\
\hline E3 & Inflamación que se extiende proximal al ángulo esplénico \\
\hline
\end{tabular}

Cuadro 5. Clasificación de la actividad de la colitis ulcerativa

Los derivados del ácido 5 aminosalicílico (5-ASA) son los medicamentos de primera línea.

\begin{tabular}{lllll}
\hline Variable & Remisión & Leve & Moderada/Grave & Fulminante \\
\hline $\begin{array}{l}\text { Número de } \\
\text { deposiciones/día }\end{array}$ & Heces formadas & $<4$ & $>6$ & $>10$ \\
\hline Sangre en heces & No & Intermitente & Frecuente & Continuo \\
\hline Urgencia & No & Ocasional & Frecuente & Continuo \\
\hline Hemoglobina & Normal & Normal & $<75 \%$ de lo normal & $\begin{array}{l}\text { Requiere } \\
\text { transfusión }\end{array}$ \\
\hline VSG & $<30$ & $<30$ & $>30$ & $>30$ \\
\hline PCR & Normal & Elevada & Elevada & Elevada \\
\hline $\begin{array}{l}\text { Calprotectina fecal } \\
\text { (mcg/g) }\end{array}$ & $<150-200$ & $>150-200$ & $>150-200$ & $>150-200$ \\
\hline $\begin{array}{l}\text { Puntaje endoscópico } \\
\text { Mayo }\end{array}$ & $0-1$ & 1 & $2-3$ & 3 \\
\hline \begin{tabular}{l} 
UCEIS \\
\hline
\end{tabular} & $0-1$ & $2-4$ & $5-8$ & $7-8$ \\
\hline
\end{tabular}

VSG: velocidad de sedimentación globular; PCR: proteína C reactiva; UCEIS: índice de gravedad endoscópica de la colitis ulcerativa. Adaptado de Referencia (13)

\section{Tratamiento}

Una vez hecho el diagnóstico y determinada la extensión y gravedad de la enfermedad, lo siguiente será establecer el tratamiento óptimo para inducir y mantener la remisión clínica (cese de sangrado rectal y normalización del hábito intestinal) y endoscópica (puntaje endoscópico Mayo 0-1), con miras a mejorar la calidad de vida, disminuir el riesgo de colectomía y de cáncer colorrectal (19).

\section{Enfermedad leve a moderada}

Los derivados del ácido 5 aminosalićlico (5-ASA) son los medicamentos de primera línea. Entre ellos, la mesalazina es el único disponible en el país. Para inducir remisión en pacientes con proctitis se inicia tratamiento con supositorios a dosis de 1g/día (20). 
En quienes no responden a la terapia con 5-ASA se recomienda adicionar corticoesteroide oral para inducir la remisión.
En colitis izquierda y extensa, donde el tratamiento tópico no tiene adecuada cobertura en la mucosa colónica, se recomienda el uso combinado de 5-ASA tópico y oral, siendo la combinación superior a la monoterapia (21). La dosis tópica recomendada es de 1 a $4 \mathrm{~g} /$ día, mientras que la dosis oral es de al menos $2 \mathrm{~g} /$ día y puede titularse hasta 4,8 g/día. El régimen de administración una vez al día es tan efectivo como el de dosis dividida y se asocia con mayor adherencia (22). La respuesta generalmente se percibirá en 14 días, pero pudiese tomar hasta dos meses para lograrse remisión clínica completa. Posteriormente, el tratamiento de mantenimiento con 5-ASA en supositorios o enemas se puede espaciar cada dos a tres días (14). La presentación oral debe continuarse diariamente (23). Por su potencial nefrotóxico se recomienda medir la función renal previo al inicio de 5-ASA, a los tres meses y luego cada año (24).

En quienes no responden a la terapia con 5-ASA se recomienda adicionar corticoesteroide oral para inducir la remisión. Las guías del Colegio Americano y la Sociedad Británica de Gastroenterología recomiendan budesonida $9 \mathrm{mg} /$ día, porque ofrece tasas más altas de cicatrización endoscópica comparada con placebo y menor porcentaje de efectos adversos sistémicos que los esteroides convencionales $(14,24,25)$.

Otra opción es usar prednisolona 40-60 mg/día. La respuesta clínica se espera en las primeras dos semanas del tratamiento. El desmonte debe ocurrir en seis a ocho semanas. Por su parte, el tratamiento de mantenimiento puede ser solo con 5-ASA si la actividad de la enfermedad es leve, se trata de una colitis ulcerativa de novo o es la primera vez que se usa 5-ASA. En pacientes con factores de mal pronóstico (edad menor de 40 años al diagnóstico, compromiso endoscópico severo, colitis extensa, PCR elevada, hipoalbuminemia, hospitalización por actividad de colitis) se debe iniciar mantenimiento con tiopurinas o biológicos $(4,14)$.

\section{Enfermedad moderada o grave}

En los pacientes con enfermedad moderada a grave y que no logren remisión con esteroides sistémicos en dos a cuatro semanas, se hará la inducción y el mantenimiento con biológicos tipo anti-TNF-a (infliximab, adalimumab o golimumab), anti-integrina (vedolizumab) o inhibidor janus quinasa (tofacitinib). En aquellos refractarios a esta terapia, se considerará el trasplante de microbiota de materia fecal o colectomía electiva $(4,14,24)$.

\section{Enfermedad aguda grave}

La enfermedad aguda grave se define como la presencia de más de seis deposiciones sanguinolentas por día y al menos un hallazgo de toxicidad sistémica (fiebre, taquicardia, hemoglobina $<10,5 \mathrm{~g} / \mathrm{dl}$ o PCR $>30 \mathrm{mg} / \mathrm{l}$ ) (26). Es una emergencia y debe tratarse agresivamente. La terapia de primera línea son los corticosteroides intravenosos (hidrocortisona $100 \mathrm{mg}$ cada seis horas o metilprednisolona $60 \mathrm{mg}$ día) por tres a cinco días (4). El $65 \%$ de los pacientes responderá y para los que persisten sintomáticos se debe adicionar ciclosporina o infliximab, ambos igual de eficaces (4). Si no hay respuesta (menos de cuatro deposiciones/día y ausencia de sagrado rectal) debe realizarse colectomía. El retraso en la cirugía aumenta el riesgo de mortalidad después de siete días (4) Otras indicaciones de colectomía son: hemorragia no controlada, perforación, carcinoma colorrectal o lesiones displásicas no susceptibles de extirpación endoscópica. La cirugía comúnmente realizada es la proctocolectomía restauradora con reservorio ileoanal (4). 


\section{Probióticos}

Si bien la evidencia es de baja calidad, y aún no se recomienda su uso sistemático, recientemente se evidenció el potencial beneficio del uso de la cepa VSL\#3 (probiótico) para inducir remisión en pacientes con colitis ulcerativa (26).

\section{Conclusión}

La colitis ulcerativa es una enfermedad con una prevalencia significativa, una incidencia en aumento y un impacto importante en la morbimortalidad. Un diagnóstico certero y un tratamiento oportuno harán la diferencia. El conocimiento de conceptos esenciales de esta enfermedad es indispensable.

\section{Conflicto de interés}

Ninguno

\section{Bibliografía}

1. Feldman M, Friedman LS, Brandt LJ. Sleisenger and Fordtran's Gastrointestinal and Liver Disease. Elsevier health sciences. 10th ed.2018; pp 2030-2031

2. Kobayashi T, Siegmund B, Le Berre C, Wei S, Ferrante M, Shen B et al. Ulcerative colitis. Nature Reviews Disease Primers. 2020;6(1).

3. Matsuoka K, Kobayashi T, Ueno F, Matsui T, Hirai F, Inoue N et al. Evidence-based clinical practice guidelines for inflammatory bowel disease. Journal of Gastroenterology. 2018;53(3):305-353.

4. Gajendran M, Loganathan P, Jimenez G, Catinella AP, Ng N, Umapathy C, Ziade N, Hashash JG. A comprehensive review and update on ulcerative colitis. Dis Mon. 2019 Dec;65(12):100851. doi: 10.1016/j.disamonth.2019.02.004. Epub 2019 Mar 2. PMID: 30837080.

5. Calvet X, Argüelles-Arias F, López-Sanromán A, Cea-Calvo L, Juliá B, De Santos $C R$, et al. Patients' perceptions of the impact of ulcerative colitis on social and professional life: Results from the UC-LIFE survey of outpatient clinics in Spain. Patient Prefer Adherence 2018;12:1815-23.

6. Lichtenstein GR, Shahabi A, Seabury SA, Lakdawalla DN, Espinosa OD, Green S, et al. Lifetime economic burden of Crohn's Disease and Ulcerative Colitis by age at diagnosis. Clin Gastroenterol Hepatol. 2020;18 (4):889-897.e10.

7. Guan Q. A Comprehensive Review and Update on the Pathogenesis of Inflammatory Bowel Disease. J Immunol Res. 2019 Dec 1;2019:7247238. doi: 10.1155/2019/7247238. PMID: 31886308; PMCID: PMC6914932.

8. Ungaro R, Mehandru S, Allen PB, Peyrin-Biroulet L, Colombel JF. Ulcerative colitis. The Lancet 2017 p. 1756-70.

9. Langholz E, Munkholm P, Davidsen M, Binder V. Course of ulcerative colitis: Analysis of changes in disease activity over years. Gastroenterology 1994;107 (1):3-11. 
10. Levine JS, Burakoff R. Extraintestinal manifestations of inflammatory bowel disease. Gastroenterol Hepatol (N Y) 2011;7 (4):235-41.

11. Danovitch SH. Fulminant colitis and toxic megacolon. Gastroenterol Clin North Am. 1989;18 (1):73-82.

12. Desai J, Elnaggar M, Hanfy A, Doshi R. Toxic Megacolon: Background, pathophysiology, management challenges and solutions. 2020.

13. Lutgens MWMD, van Oijen MGH, van der Heijden GJMG, Vleggaar FP, Siersema $P D$, Oldenburg B. Declining risk of colorectal cancer in inflammatory bowel disease: an updated meta-analysis of population-based cohort studies. Inflamm Bowel Dis 2013;19 (4):789-99.

14. Rubin DT, Ananthakrishnan AN, Siegel CA, Sauer BG, Long MD. ACG Clinical Guideline: Ulcerative Colitis in Adults. Am J Gastroenterol 2019;114 (3):384-413.

15. Bentley E, Jenkins D, Campbell F, Warren B. How could pathologists improve the initial diagnosis of colitis? Evidence from an international workshop. J Clin Pathol 2002;55 (12):955-60.

16. Sherwood R, Walsham N. Fecal calprotectin in inflammatory bowel disease. Clinical and Experimental Gastroenterology. 2016:21.

17. Mao R, Xiao YL, Gao X, Chen BL, He Y, Yang L, et al. Fecal calprotectin in predicting relapse of inflammatory bowel diseases: A meta-analysis of prospective studies. Inflamm Bowel Dis 2012;18 (10):1894-9.

18. Panes J, Bouhnik Y, Reinisch W, Stoker J, Taylor SA, Baumgart DC, et al. Imaging techniques for assessment of inflammatory bowel disease: Joint ECCO and ESGAR evidence-based consensus guidelines. J Crohn's Colitis 2013;7 (7):556-85.

19. Peyrin-Biroulet L, Sandborn W, Sands BE, Reinisch W, Bemelman W, Bryant R V., et al. Selecting Therapeutic targets in inflammatory bowel disease (STRIDE): Determining Therapeutic goals for treat-to-target. Am J Gastroenterol. 2015;110 (9):1324-38.

20. Marshall JK, Thabane M, Steinhart AH, Newman JR, Anand A, Irvine EJ. Rectal 5 -aminosalicylic acid for induction of remission in ulcerative colitis. Cochrane Database Syst Rev. 2010; (1).

21. Ford AC, Khan KJ, Achkar JP, Moayyedi P. Efficacy of oral vs. Topical, or combined oral and topical 5-aminosalicylates, in ulcerative colitis: Systematic review and meta-analysis. Am J Gastroenterol; 2012 p. 167-76.

22. Kane S V., Cohen RD, Aikens JE, Hanauer SB. Prevalence of nonadherence with maintenance mesalamine in quiescent ulcerative colitis. Am J Gastroenterol 2001;96 (10):2929-33.

23. Bressler B, Marshall JK, Bernstein CN, Bitton A, Jones J, Leontiadis GI, et al. Clinical practice guidelines for the medical management of nonhospitalized ulcerative colitis: The Toronto consensus. Gastroenterology 2015;148 (5):1035-1058.e3. 
24. Lamb CA, Kennedy NA, Raine T, Hendy PA, Smith PJ, Limdi JK, Hayee B, Lomer MCE, Parkes GC, Selinger C, Barrett KJ, Davies RJ, Bennett C, Gittens S, Dunlop MG, Faiz O, Fraser A, Garrick V, Johnston PD, Parkes M, Sanderson J, Terry H; IBD guidelines eDelphi consensus group, Gaya DR, Iqbal TH, Taylor SA, Smith M, Brookes M, Hansen R, Hawthorne AB. British Society of Gastroenterology consensus guidelines on the management of inflammatory bowel disease in adults. Gut. 2019 Dec;68(Suppl 3):s1-s106. doi: 10.1136/gutjnl-2019-318484. Epub 2019 Sep 27. Available from: https://pubmed.ncbi.nlm.nih.gov/31562236/

25. Rubin DT, Cohen RD, Sandborn WJ, Lichtenstein GR, Axler J, Riddell RH, Zhu C, Barrett AC, Bortey E, Forbes WP. Budesonide Multimatrix Is Efficacious for Mesalamine-refractory, Mild to Moderate Ulcerative Colitis: A Randomised, Placebo-controlled Trial. J Crohns Colitis. 2017 Jul 1;11(7):785-791. doi: 10.1093/ ecco-jcc/jjx032. Erratum in: J Crohns Colitis. 2017 Dec 4;11(12):1510.

26. Astó E, Méndez I, Audivert S, Farran-Codina A, Espadaler J. The Efficacy of Probiotics, Prebiotic Inulin-Type Fructans, and Synbiotics in Human Ulcerative Colitis: A Systematic Review and Meta-Analysis. Nutrients. 2019 Jan 30;11(2):293. doi: 10.3390/nu11020293. 